\title{
Coartación de aorta: diagnóstico de sospecha en la consulta de Atención Primaria
}

\author{
N. M. P uente García, M. L. Fernández Gómez, D. Voces García \\ Médicos Especialistas en Medicina Familiar y Comunitaria. \\ C.S. El Greco. Getafe. ${ }^{*}$ C.S. Mendiguchía Carriche. Leganés. Madrid
}

\section{RESUMEN}

La coartación aórtica es un estrechamiento de la luz aórtica por debajo de la salida de la subclavia izquierda que se caracteriza por hipertensión arterial en miembros superiores y debilidad de pulsos femorales. En el examen físico puede auscultarse un soplo sistólico continuo en la espalda. Existen además alteraciones radiológicas y electrocardiográficas características. El diagnóstico se confirma mediante ecocardiografía y arteriografía.

Nosotros presentamos un caso clínico visto en nuestra consulta de Atención Primaria.

Palabras clave: Coartación de aorta. Hipertensión arterial. Cardiopatía congénita.
Coarctation of the aorta: diagnosis in Primary Health Care

\begin{abstract}
Coartation of the aorta typically consits of a discrete ridge extending into the aortic lumen just distal to the left subclavian artery. This condition results in hypertension in the arms and the femoral arterial pulses are weak and delayed. On physical examination a harsh systolic ejection murmur may be identified along the left sternal border and in the back, particularly over the coartation. On the chest radiograph and on the electrocardiogram we meet typical changes. The coarctation may be visualized echocardiographically and aortography.

We report a clinical case we have seen in our Primary Health Care's room.
\end{abstract}

Key words: Aortic coarctation. Hypertension. Heart defects. Congenital.

\section{INTRODUCCIÓN}

La coartación de aorta es una enfermedad caracterizada clínicamente por hipertensión arterial y a pesar de su escasa incidencia debe ser tenida en cuenta en el diagnóstico diferencial de hipertensión arterial secundaria ya que puede establecerse un diagnóstico de presunción a partir de la historia clínica y la exploración física.

\section{CASO CLÍNICO}

Se trata de un varón de 16 años que acude de urgencia a nuestra consulta por epístaxis de dos horas de duración, junto con sensación de mareo sin giro de objetos.

No refiere antecedentes familiares de interés.

En cuanto a sus antecedentes personales destaca una cirugía de ectopia testicular a los 6 años, varicela a los 4 años y disminución de agudeza auditiva desde hace un año en oído derecho.

En la anamnesis, sufre episodios de sangrado nasal desde hace años que han aumentado en frecuencia en las últimas semanas y que siempre cedían espontáneamente. Desde hace un año presenta calambres nocturnos en las piernas, claudicación de miembros inferiores cuando hace ejercicio, sobre todo al correr, a veces asociado a dolor en el cuá- 
driceps que desaparecía en reposo y mareos en relación a grandes esfuerzos, que le obligaban a dejar la actividad física con lo que desaparecían. El resto de la anamnesis era normal.

En la exploración física vemos un paciente de constitución atlética, con buen estado general, normocoloreado, hidratado y perfundido. Afebril, con tensión arterial de 230/135 $\mathrm{mmHg}$ en brazos. Cabeza y cuello: pupilas isocóricas y normorreactivas, latido visible en región supraesternal, carótidas rítmicas con soplo sistólico bilateral, la presión venosa yugular era normal. Tórax: se palpaba circulación colateral a nivel de espacios intercostales superiores. Auscultación cardiaca: latía rítmico a 90 latidos por minuto, se auscultaba soplo sistólico en foco aórtico y de menor intensidad en foco mitral con refuerzo del segundo tono. Auscultación pulmonar: se apreciaba un murmullo vesicular normal, con un soplo continuo, en tercio superior de espalda a nivel interescapular, más intenso en hemitórax izquierdo. La exploración del abdomen era normal. Extremidades: los pulsos femorales eran simétricos pero menos intensos que los humerales y radiales, la tensión arterial sistólica en miembros inferiores era de $140 \mathrm{mmHg}$. El resto de la exploración física estaba dentro de lo normal.

En cuanto a pruebas complementarias, se pidieron electrocardiograma, radiografía de tórax y analítica de urgencia. La bioquímica básica, hemograma y orina fueron normales; en el electrocardiograma (Fig. 1) se observaban signos de hipertrofia de ventrículo izquierdo y la radiografía de tórax (Fig. 2) mostraba crecimiento de ventrículo izquierdo, dilatación aórtica y muescas en rebordes inferiores de algunos arcos costales.

Ante el hallazgo en un varón joven de: hipertensión arterial, claudicación de miembros inferiores con el ejercicio, diferencia de presiones sistólicas de miembros superiores e inferiores de más de $30 \mathrm{mmHg}$, debilidad de pulsos femorales con respecto a humeral y radial, soplo sistólico en la espalda, se sospechó una coartación de aorta y se decidió derivar urgente a cardiología, donde confirmaron el diagnóstico tras la realización de ecocardiograma (Fig. 3) y arteriografía (Fig. 4) que ponían de manifiesto una coartación de aorta de tipo del adulto, postsubclavia izquierda con hipertrofia de arterias mamarias y de los troncos tirocervicales.

El tratamiento fue quirúrgico, por el servicio de cirugía cardiovascular, realizando una ampliación de aorta con un parche romboidal de Dacron Woven en la zona de la estenosis; inicialmente también precisó tratamiento farmacológico para control de la hipertensión, que unos meses más tarde se retiró por quedar totalmente asintomático.

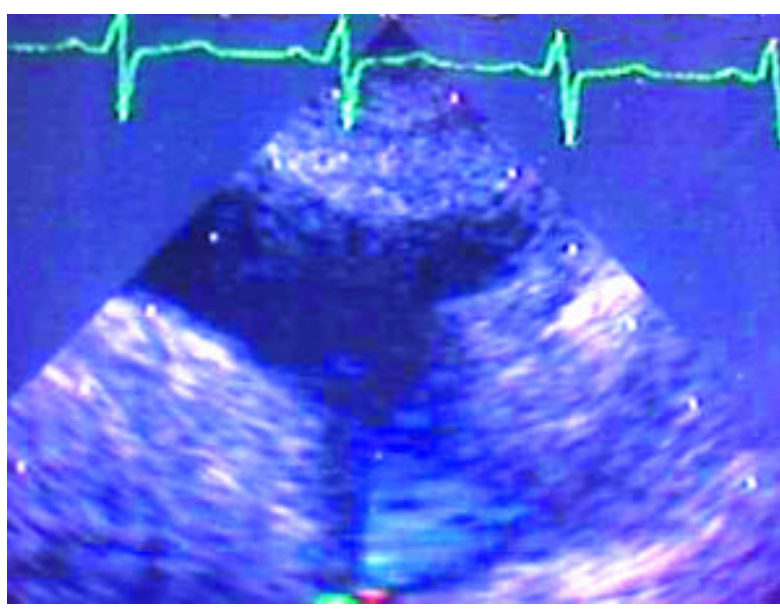

Figura 1

Rx Tórax: hipertrofia de ventrículo izquierdo, muescas costales

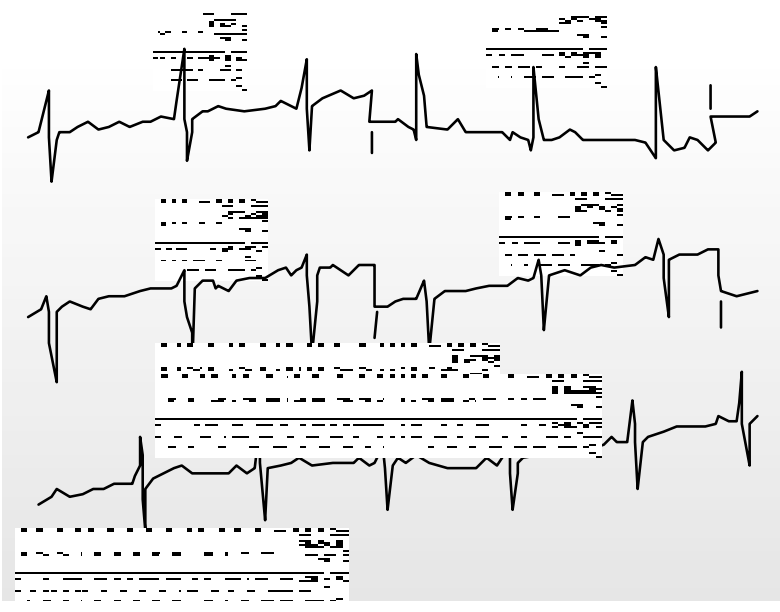

Figura 2a

EKG: eje desviado a la izquierda

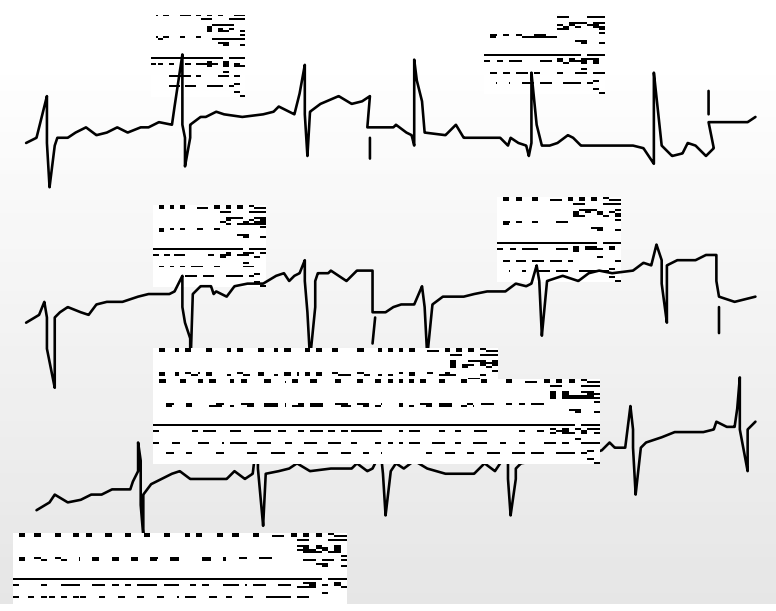

Figura $2 b$

EKG: eje desviado a la izquierda 


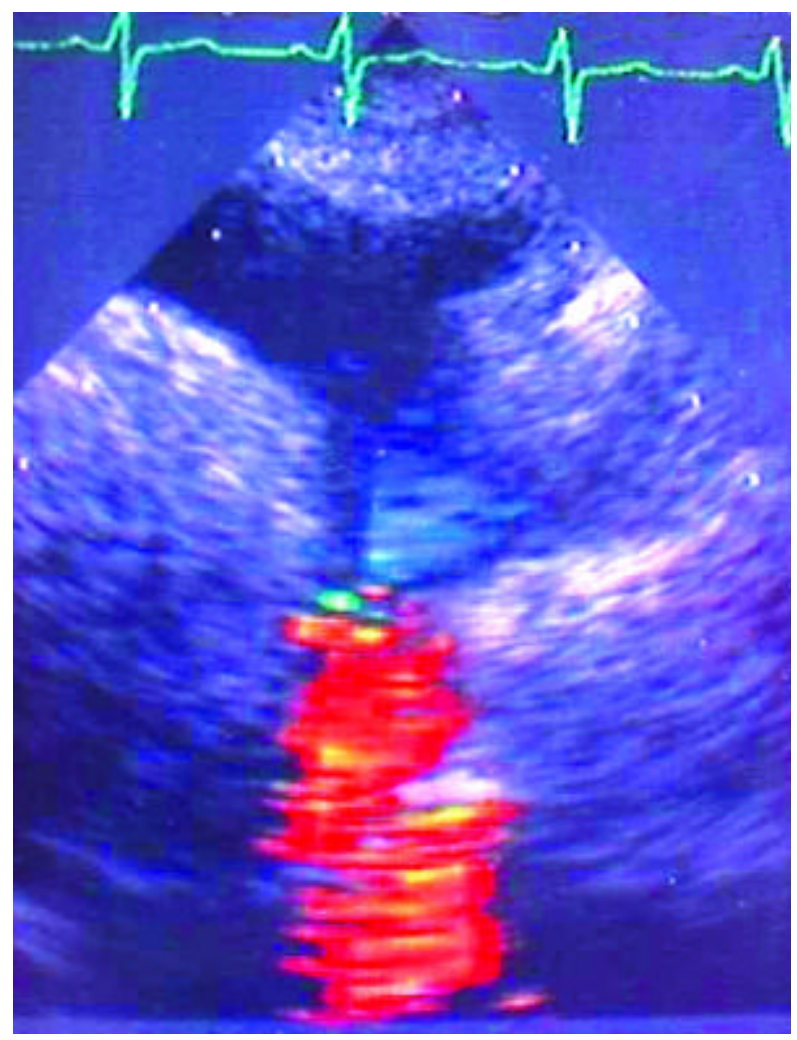

Figura 3

Ecocardiograma con doppler

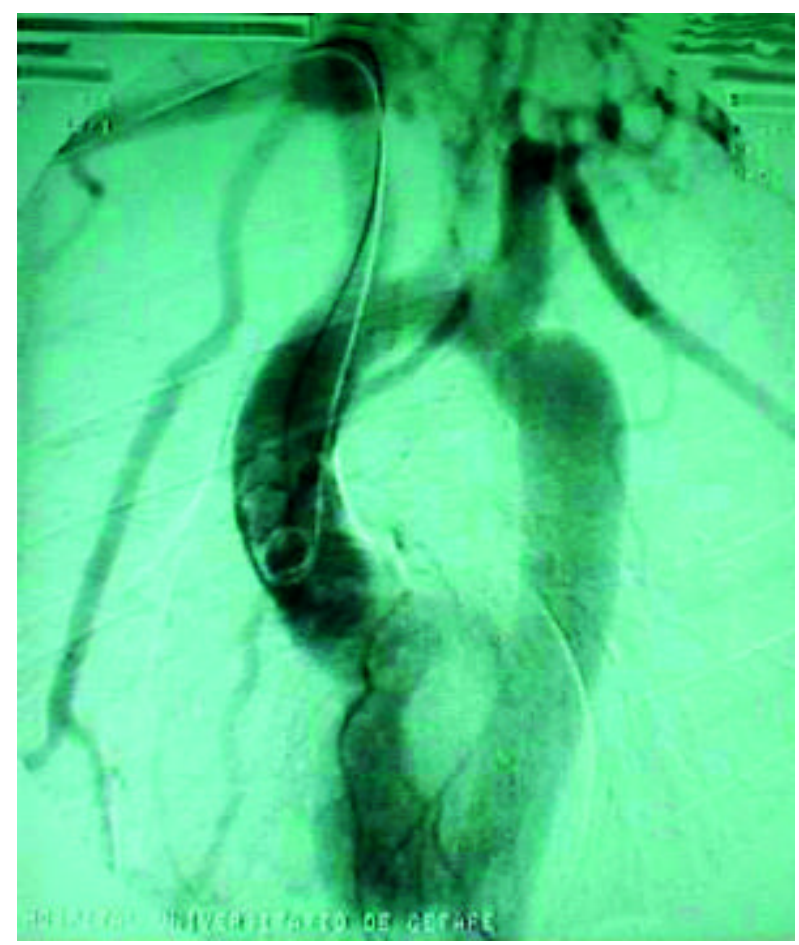

Figura 4

Cateterismo

\section{DISCUSIÓN}

La coartación aórtica consiste en un estrechamiento de la luz aórtica, generalmente a nivel de la unión del cayado aórtico con la aorta descendente, por debajo del nacimiento de la arteria subclavia izquierda, a nivel o justo debajo de la inserción del ligamento arterioso. La base anatómica de la alteración consiste en una deformidad de la pared media de la arteria manifestada por un pliegue que estrecha de forma concéntrica la pared aórtica. Esta constricción produce una disminución del flujo sanguíneo hacia la parte inferior del organismo de forma que los pulsos y la presión son mayores en los brazos que en las piernas.

Menos comúnmente la coartación se localiza inmediatamente proximal a la arteria subclavia izquierda en cuyo caso se nota diferencia de la presión y los pulsos entre ambos brazos.

\section{Epidemiología}

Las cardiopatías congénitas se encuentran en un $1 \%$ de los recién nacidos vivos y de ellas un 5\% corresponden a la coartación de aorta, ocupando un lugar destacado. En las autopsias se encuentra, en aproximadamente 1:1550 pacientes ${ }^{1}$. Es de 2 a 5 veces más frecuente en varones que en mujeres ${ }^{2}$. Aunque la coartación suele presentarse como hecho aislado puede asociarse en ocasiones a otras anomalías, siendo las más frecuentes: la válvula aórtica bicúspide (20-40\%), defectos del septo ventricular, ductus arterioso permeable, estenosis mitral, cardiopatía coronaria debida a la hipertensión, riñón poliquístico, aneurisma congénito de las arterías del polígono de Willis y disgenesias gonadales como el síndrome de Turner ${ }^{3}$.

\section{Clínica}

La mayoría de los niños permanecen asintomáticos y la enfermedad puede pasar desapercibida hasta bien entrada la edad adulta. Clásicamente la edad típica de presentación de los síntomas es entre los veinte y treinta años aunque en la mayoría de los sujetos el diagnóstico se realiza durante exámenes físicos rutinarios cuando se observa hipertensión arterial sistólica en los brazos, con ausencia o disminución de pulsos femorales. Cuando los síntomas se presentan, suelen estar relacionados con la hipertensión: cefalea, epístaxis, mareo, palpitaciones, frialdad y claudicación con el ejercicio en miembros inferiores. A veces los pacientes consultan por tener síntomas de fallo cardiaco o disección aórtica. Las mujeres con coartación aórtica tienen mayor riesgo de disección aórtica durante el embarazo ${ }^{2}$. 


\section{Diagnóstico}

En el examen físico se observa típicamente que la tensión arterial sistólica es mayor en los brazos que en las piernas, mientras que las tensiones diastólicas son similares, así como una presión aumentada en el pulso radial. Los pulsos de la arteria femoral están retrasados y son débiles. La inspección puede sugerir el diagnóstico al observar un latido arterial visible en el hueco supraesternal. Las extremidades superiores y el tórax pueden estar más desarrolladas que las inferiores. La circulación colateral por los vasos dilatados se palpa en los es pacios intercostales, en la axila o en la región interescapular. Se ausculta un soplo mesosistólico en la parte anterior del tórax, espalda y apófisis espinosas que puede transformarse en continuo si la luz está lo bastante estenosada para producir un chorro a alta velocidad durante todo el ciclo cardiaco. Un clic sistólico de eyección debido a una válvula aórtica bicúspide puede oírse con frecuencia y el segundo ruido cardiaco está acentuado. Un murmullo sistólico causado por el flujo aumentado por los vasos colaterales a través de la arteria torácica interna, intercostal, subclavia y arterias escapulares también puede oírse en la espalda².

En la radiografía de tórax el aumento de flujo colateral a través de las arterias intercostales produce muescas generalmente simétricas en la cara inferior de los arcos costales posteriores de la $3^{\mathrm{a}}$ a la $8^{\text {a }}$ costilla. Las muescas no se ven en las costillas anteriores porque la arteria intercostal anterior no se localiza en los surcos costales. La coartación puede ser visible como una escotadura de la aorta en el lugar de la coartación y puede verse una dilatación pre y otra postestenótica produciendo el signo del " 3 ".

En el electrocardiograma es típico observar una desviación del eje eléctrico a la izquierda junto con una hipertrofia ventricular izquierda.

Para el diagnóstico de confirmación se utilizan distintas técnicas.

El ecocardiograma con doppler nos permite estimar el gradiente de presión transcoartación. Para obtener información anatómica sobre la localización y dimensiones de la coartación se utilizan habitualmente la tomografía axial computerizada, la resonancia magnética y la arteriografía. Esta última nos permite además observar la circulación colateral ${ }^{3}$.

\section{Complicaciones}

Las complicaciones de la coartación aórtica incluyen hipertensión, fallo ventricular izquierdo, disección aórtica debida a necrosis quística de la media, enfermedad coronaria prematura, endocarditis infecciosa localizada en la coartación o sobre la válvula aórtica bicúspide asociada y accidentes cerebrovasculares, debidos a ruptura de aneurisma intracerebral congénito ${ }^{4,5}$.

\section{Tratamiento}

La cirugía reparadora es el único tratamiento definitivo de la coartación de la aorta y generalmente los resultados son buenos. La reparación quirúrgica debería considerarse en pacientes con un gradiente de presión transcoartación de más de $30 \mathrm{mmHg}^{3}$. Aunque en la dilatación con balón en una terapia alternativa, el procedimiento se asocia con una mayor incidencia de aneurisma aórtico y recurrencia de la coartación que la reparación quirúrgica. Ésta consiste en la resección del segmento estenótico y anastomosis término-terminal o la sustitución del segmento aórtico afectado por una prótesis ${ }^{6}$.

En ausencia de reparación quirúrgica más del 90\% de los pacientes con coartación mueren antes de los 50 años de enfermedades cardio o cerebrovasculares. El seguimiento más largo realizado de supervivientes después de la cirugía muestra una probabilidad de supervivencia de más del $70 \%$ a los 40 años de la cirugía ${ }^{4}$.

Entre las complicaciones postquirúrgicas se incluye hipertensión residual o recurrente, recurrencia de la coartación y las posibles secuelas de una válvula aórtica bicúspide.

La hipertensión sistémica postoperatoria, en ausencia de coartación residual, parece estar en relación con la duración de la hipertensión antes de la intervención, ocurre en un $27 \%^{1}$ de los pacientes después de la cirugía y aumenta su incidencia con la duración del seguimiento.

Se relaciona con la edad a la que se intervino, y cuando la intervención se realiza de los dos a los nueve años el $90 \%$ permanece normotenso 5 años después y el 25\%, 25 años después. En contraste, aquellos que recibieron cirugía después de la edad de 40 años el $50 \%$ presentan hipertensión y muchos de ellos con una presión normal después de la cirugía tienen una respuesta hipertensiva al ejercicio que aumenta su morbimortalidad cardio y cerebrovascular ${ }^{3,4}$.

La recoartación ocurre en aproximadamente un $40 \%$ de los supervivientes a los 16 años de la intervención; esta complicación se relaciona con la resección incompleta de la lesión (coartación residual), fallo de crecimiento en el lugar de la anastomosis (coartación recurrente) o a una combinación de ambos. Un aumento de la incidencia de recoartación se espera durante el seguimiento a largo plazo. La precocidad de la intervención (menor de dos años) y el bajo peso son factores de riesgo independientes para la recoartación, que en muchas 
ocasiones puede ser tratada con éxito mediante la dilatación percutánea con balón.

La enfermedad de la válvula aórtica significativa ocurre en un $37 \%$ de sujetos intervenidos en la infancia y en un $58 \%$ de sujetos adultos operados y puede conllevar la necesidad de sustitución de la válvula aórtica en una fracción significativa de estos supervivientes. En varias series de pacientes, éstos fallecen por enfermedad de la válvula aórtica o por complicaciones derivadas de la sustitución de la misma ${ }^{4}$.

La supervivencia después de la intervención de la coartación está influida por la edad del paciente en el momento de la cirugía. Después de la inter vención durante la infancia un $89 \%$ de pacientes viven 15 años después y un $83 \%$ continúan vivos 25 años después.

La tasa de supervivencia va disminuyendo con la edad y cuando se interviene a pacientes mayores de 40 años, la supervivencia a los 15 años es solo del $50 \%{ }^{3,7}$.

\section{Seguimiento del paciente desde Atención Primaria}

El electrocardiograma y la radiología de tórax resultan útiles pero no específicos para la información diagnóstica y pronóstica. El electrocardiograma es normal en un $24-48 \%$ de los casos y las anomalías más frecuentes suelen ser bloqueos de rama izquierda o derecha o isquemia. La hipertrofia ventricular izquierda se encuentra en más del $27 \%$ de los casos, y en muchas ocasiones se asocia a valvulopatía aórtica u otras patologías como recoartación, insuficiencia mitral, defecto ventrículo septal, etc.
El screening regular y el tratamiento agresivo para la hipertensión en los grupos de riesgos son esenciales. El gradiente de presión entre los miembros superiores e inferiores deberá ser revisado en cada consulta y establecerse una derivación por sospecha de recoartación si es mayor de $20 \mathrm{mmHg}$.

En ocasiones, es necesaria la modificación del riego cardiovascular, y el consejo para el uso de profilaxis antibiótica para los procesos dentales y otros procesos ${ }^{1,8}$.

Aunque la coartación aórtica es una enfermedad poco frecuente, debe ser recordada en Atención Primaría, bien sea en las consultas de pediatría, con motivo de las revisiones sistemáticas, bien sea en el diagnóstico diferencial de la hipertensión arterial del adulto joven.

Uno de los principales roles para el médico de familia será ayudar a los padres a poner el diagnóstico en perspectiva, clarificando expectativas y errores de concepto ${ }^{8,9}$.

\section{CORRESPONDENCIA:}

N. M. Puente García

C/ Penélope, 4

28230 Las Rozas. Madrid

\section{Bibliografía}

1. Jenkins NP, Ward C. Coarctation of the aorta: natural history and outcome after surgical treatment. QJM 1999; 92 (7): 365-71.

2. Brickner ME, Hillis LD, Lange RA. Congenital heart disease in adults. N Engl J Med 2000; 27; 342 (4): 256-63.

3. Navarro-López F. Cardiopatías Congénitas. En: Farreras-Rozman. Tratado de medicina interna (13 ed.). Madrid, 1995; 597-8.

4. Findlow J. Congenital hearth disease in adults. $\mathrm{Br} \mathrm{J}$ Anaesth 1997; 78 (4): 416-30.

5. Friedman W, Child J. Cardiopatías congénitas en el adulto. En: Harrison, Principios de medicina interna (14 $4^{\mathrm{a}}$ ed.) Madrid, 2000; $1492-93$.

6. McCrindle BW.Coarctation of the aorta. Curr Opin Cardiol 1999; 14 (5): 448-52.

7. Rothman A. Interventional therapy for coarctation of the aorta. Curr Opin Cardiol 1998; 13 (1): 6-72.

8. Saenz RB, Beebe DK, Triplett LC. Caring for infants with congenital heart disease and their families. Am Fam Physician 1999; 59 (7): 1857-68.

9. Swan L, Wilson N, Houston AB, Doig W, Pollock JC, Hillis WS. The long- term management of the patient with an aortic coarctation repair. Eur Heart J 1998; 19 